\title{
FAIR: The GSI future project
}

\author{
Carsten Schwarz ${ }^{a}$ \\ ${ }^{a}$ GSI, Planckstrasse 1, 64291 Darmstadt, Germany
}

\begin{abstract}
The Gesellschaft für Schwerionenforschung (GSI) plans a major new international research facility at Darmstadt, Germany. The principal goal of the new facility is to provide the European science community with a worldwide unique and technically innovative accelerator system to perform future forefront research in the sciences concerned with the basic structure of matter. The facility will provide an extensive range of particle beams from protons and their antimatter partners, antiprotons, to ion beams of all chemical elements up to the heaviest one, uranium, with in many respects world record intensities. The proposed facility consists of a 100/300 Tm double-ring synchrotron SIS100/300 and a system of associated storage rings for beam collection, cooling, and experimentation. The heart of the new facility, the double-ring synchrotron, provides for fast acceleration utilizing novel, rapidly cycling super-conduction magnets. These and the usage of stochastic and electron cooling of the beams are the key features of the new facility.
\end{abstract}

\section{Introduction}

The proposed project FAIR (Facility for Antiproton and Ion Research) is an international accelerator facility of the next generation. It builds on the experience and technological developments already made at the existing GSI facility, and incorporates new technological concepts. At its heart is a double ring facility with five times the circumference of the current SIS. A system of cooler-storage rings for effective beam cooling at high energies and various experimental halls will be connected to the facility.

The existing GSI accelerators serve as injector for the new facility. The double-ring synchrotron will provide ion beams of unprecedented intensities as well as of considerably increased energy. Thereby intense beams of secondary beams - unstable nuclei or antiprotons - can be produced. The system of storage-cooler rings allows the quality of these secondary beams - their energy spread and emittance - to be drastically improved. Moreover, in connection with the double ring synchrotron, an efficient parallel operation of up to four scientific programs can be realized at a time. The project is based on many technological innovations, the most important of which are: 
- Highest Beam Intensities. Intensities of primary heavy-ion beams will increase by a factor of 100 to 1000, secondary radioactive beams by a factor of up to 10000 over the present GSI facility.

- Brilliant Beam Quality. Precision beams will be achieved through sophisticated beam handling, such as stochastic and electron cooling of the ion beams. Together with the statistical accuracy and sensitivity that result from high beam intensities and interaction rates, these beams will allow entering new areas of precision studies in the science fields envisioned.

- Highest Beam Energies. An increase of energy by at least a factor of 20 is planned for beams as heavy as uranium.

- Parallel Operation of the different research programs.

\section{Accelerator concept}

The research to be performed with this future facility covers experiments with primary and secondary beams of ions with highest intensity. They also include an high precision antiproton beam. The concept and layout of the new facility as shown in Fig. 1 has evolved from the scientific requirements: substantially higher intensities, compared to the present system, are achieved through faster cycling and, for heavy ions, lower charge state, which enters quadratically in the space-charge limit. The reduced charge state and a desired beam energy of up to $1.5 \mathrm{AMeV}$ for radioactive beam production, requires a larger magnetic bending power. The SIS100, the number in the abbreviation denotes the bending power in Teslameter, fulfills these aspects and is the working horse for the accelerator complex. It is injected by the existing but upgraded SIS18 of GSI with $\mathrm{U}^{+28}$, yielding 100 to 1000 times more intense beams than today. For nuclear structure and astrophysics experiments, these intense beams are focused on a production target generating radioactive beams by fragmentation. After the superconducting fragment separator (SuperFRS) beam intensities will be up to 10000 times higher than at today's GSI facility.

These radioactive beams can be cooled by bunch rotation, stochastic cooling, and electron cooling in storage rings (CR, RESR, NESR), or used directly in a slow extraction for fixed target experiments at $0-1.5 \mathrm{AGeV}$.

In-beam experiments in the New Experimental Storage Ring (NESR) allow high precision mass measurements of nuclei far from stability, the measurement of the strong interaction radius and the electromagnetic interaction radius, excited levels of these unstable nuclei through transfer reaction and inelastic scattering, and more.

Antiprotons, generated by a $30 \mathrm{GeV}$ proton beam, rapidly cooled and accumulated in the Refurbished Experimental Storage Ring (RESR) and Collector Ring (CR), will be reinjected in the SIS100 and accelerated to momenta of 1.5-15 GeV for experiments in the High Energy Storage Ring (HESR). Here, up to $10^{11}$ antiprotons are accumulated and continuously cooled by statistical and electron cooling. The electron cooling will provide 


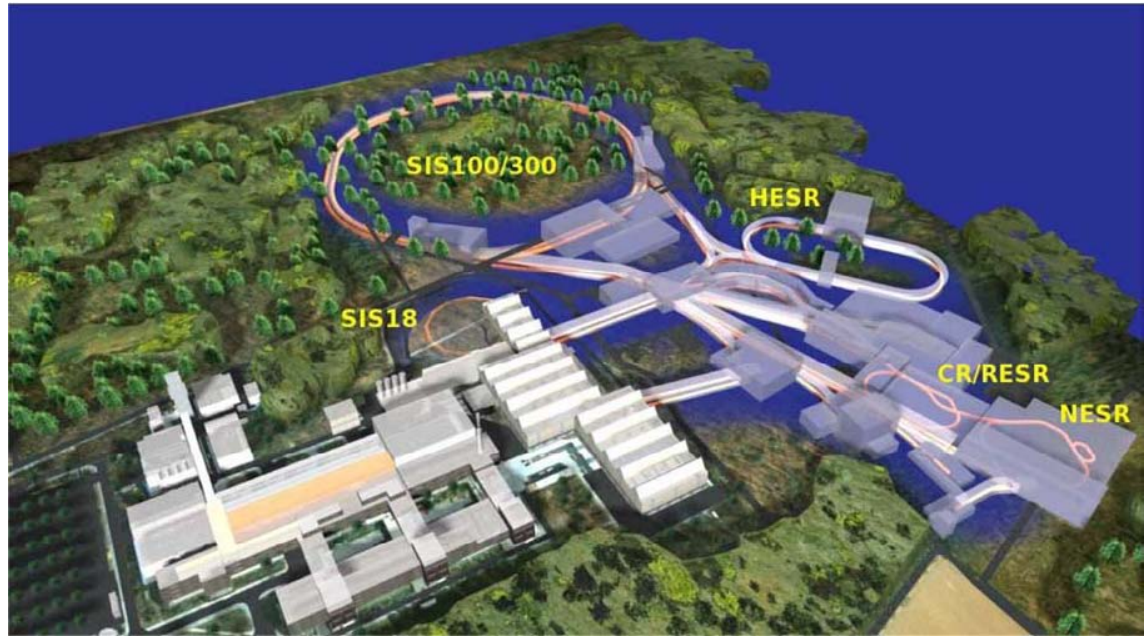

Figure 1: View of the planned Facility for Antiproton and Ion Research (FAIR).

a momentum resolution of $\delta p / p \approx 10^{-5}$. Typical collision rates of up to $2 * 10^{7}$ interaction $s^{-1}$ are foreseen, matching the production rate of the antiprotons in the facility.

When the charge-space limit in the storage rings is not so important, highly charged ions can be accelerated to relativistic energies. In SIS100 $U^{+73}$ is accelerated to $10 \mathrm{AGeV}$, stripped full to $U^{+92}$ and then transferred to the second synchrotron SIS300, which can accelerate it to energies up to $35 \mathrm{AGeV}$. A beam of $10^{10} \mathrm{U} s^{-1}$ can be slowly extracted and sent to a fixed target in the high-energy nuclear collision cave. Here also protons with energies up to $90 \mathrm{GeV}$ can be used.

An important point for the design of the facility is the high degree of parallel operation of the different research programs. A cooled and post-accelerated antiproton beam can be used in parallel to a fixed target experiment with radioactive beams and relativistic heavy-ion beams slowly extracted from the SIS 300, and an additional beam puls every few minutes can be delivered to the plasma physics research program.

\section{Research programs}

The research goal of the planned facility encompasses all aspects of hadronic matter, including the investigation of fundamental symmetries and interactions that are relevant for this regime. Further, many-body aspects play an important role in the research programs unveiling all their levels of the hierarchical structure of matter. Therefore the research programs encompass

- Investigations with beams of short-lived radioactive nuclei, addressing important 
questions about nuclei far from stability, areas of astrophysics and nucleo-synthesis in supernovae and other stellar processes, and tests of fundamental symmetries.

- The study of hadronic matter at the sub-nuclear level with beams of antiprotons, including the two key aspects: confinement of quarks and the generation of the hadron masses. They are intimately related to the existence and spontaneous breaking of chiral symmetry, a fundamental property of strong interactions.

- The study of compressed, dense hadronic matter in nucleus-nucleus collisions at high energies.

- The study of bulk matter in the high-density plasma state, a state of matter of interest for inertial confinement fusion and astrophysical settings.

- Studies of quantum electrodynamics (QED), of extremely strong electro-magnetic fields, and of ion-matter interactions.

As an example of the many experiments only one will be discussed here:

\section{PANDA experiment with antiprotons}

For the understanding of nuclear forces, the residual interaction between quarks, it is important to investigate the forces between quarks. Quantum Chromodynamics (QCD) is the theory describing such forces and has proven to be very successful at large momentum transfer, where perturbative approximations are possible. At low momentum transfer, where the QCD coupling constant is large, one enters an energy regime describing the world where we observe mesons and baryons as effective degrees of freedom. This is the regime of 'strong' QCD, where perturbation approximations fail and where are a lot of new things to learn about. The research with charm quarks might be an excellent tool, to shed light on strong QCD, to understand the quark confining and restoration of chiral symmetry. Charmonium played already in the past an important role: discovered in 1974, it is the charmonium where the parameters for the quark-quark potential are tuned. On the one hand, the coupling constant for the interaction with charm quarks $\alpha_{s} \approx 0.3$ is small enough to justify the application of perturbative approximations, on the other hand it is large enough, that nonperturbative corrections become visible.

For above reasons, one important research topic of our project will be high precision spectroscopy of the Charmonium system, in order to test and improve the predictions of Lattice Quantum Chromodynamics (LQCD), the most modern approach to this theory. Only little is known about the charmonium groundstate, $\eta_{c}$, whose width is reported as $\Gamma\left(\eta_{c}\right) \approx 24 \mathrm{MeV}$ [2] which is difficult to explain by simple quark models [3]. The scanning of resonances with an electron cooled antiproton beam having a momentum resolution of $\delta p / p \approx 10^{-5}$ and sufficient luminosity is an important experimental ingredient for the determination of the energy and width of those states, and helps to identify weak channels, when background reactions are present. This can be demonstrated by the comparison of the width of the observed $\chi_{1}$ resonance in $e^{+} e^{-}$annihilation and that in $\bar{p} p$ annihilation with a cooled antiproton beam, shown in Fig. 2. 


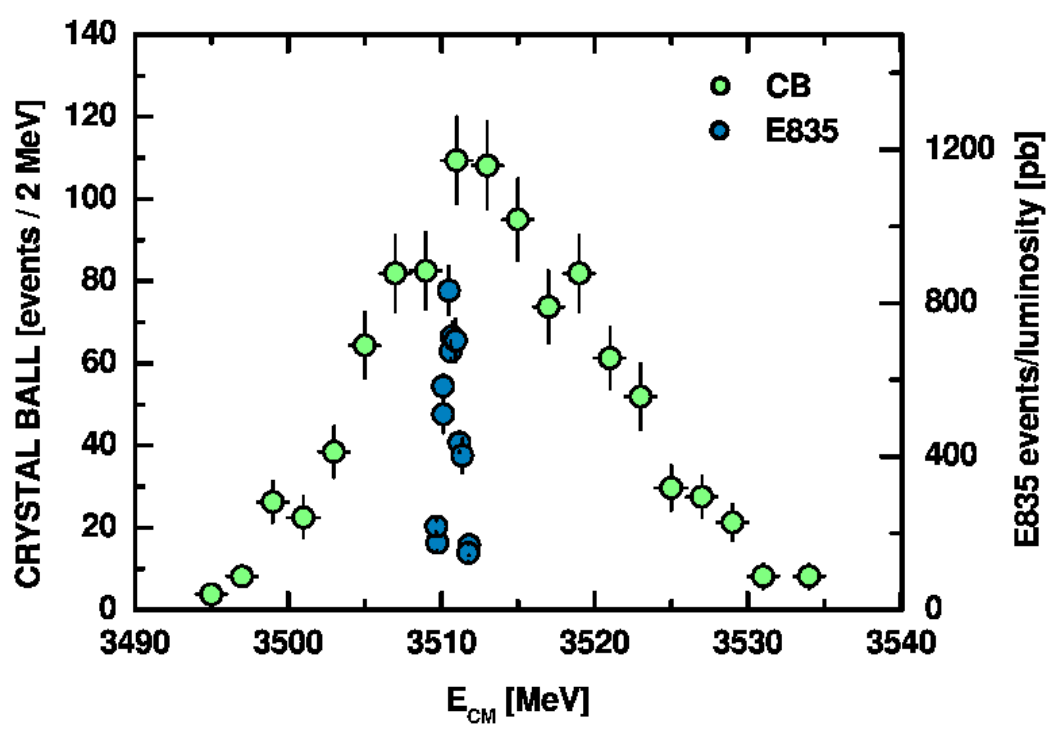

Figure 2: Comparison of measurements of the $\chi_{c 1}$ resonance obtained by $e^{+} e^{-}$annihilation (Crystal Ball, SLAC) and by $\bar{p} p$ annihilation (E835, Fermilab).

LQCD also predicts exotic objects consisting of two quarks with exited gluons (hybrids) $[6,7]$ and so called glueballs consisting only out of gluons [8]. Such exotic objects may have been already found in the light quark sector, the $\pi_{1}(1400)$ [4] and the $\pi_{1}(1600)$ [5] for hybrids and the $f_{0}(1500)$ as a candidate for a glueball. But other interpretations may still be possible. The search for such objects there is complicated by the ample presence of broad resonances of several $100 \mathrm{MeV}$ with mixing in case of identical quantum numbers. In the Charmonium sector the situation is cleaner: there are only eight narrow states giving hope to identify additional predicted states in a clean way. Experimental signatures for these objects partially are exotic quantum numbers and otherwise comparison with model predictions. Hybrid states with exotic quantum numbers cannot be produced in formation experiments. Their observation in production experiments and non-observation in formation experiments will confirm the results from partial-wave analysis.

Recently, mass shifts of pions [9] and kaons [10] in the nuclear medium were observed and are part of ongoing research at GSI for the light vector mesons $(\rho, \omega, \phi)[11]$. While for the charmonium no substantial mass shifts are expected, predictions exist that for the combination of a light quark with a charm quark, the charmed D-meson, such mass shifts account for half of the mass shift observed for kaons [12]. The experimental signature for such a mass shift would be the change of branching ratios close to the $D \bar{D}$-threshold, by 
annihilating antiprotons in nuclear targets. Further, the measurement of $J / \psi$-suppression in such annihilations yield complementary information for understanding important signals of the creation of a quark-gluon plasma [13].

A relatively old field, the investigation of hypernuclei [14] becomes revived by the upcome of high rate capable gamma detectors like VEGA or AGATA. In proton-antiproton annihilations high production rates of single or double strange baryons are expected.

\section{References}

[1] An International Accelerator Facility for Beams of Ions and Antiprotons - Conceptional Design Report, GSI 2001, http://www.gsi.de/GSI-Future/

[2] A. Tomaradze, Proceedings 9th International Conference on Hadron Spectroscopy (Hadron 2001), Protvino, Russia, August 25-September 1, 2001.

[3] M. B. Voloshin, private communication.

[4] A. Abele, J. Adomeit, C. Amsler, C.A. Baker et al., Phys. Lett. B 423 (1998) 175.

[5] J. Reinnarth, Biennial Conference on Low Energy Antiproton Physics (LEAP 2000), Venice (Italy, Aug.2000)

[6] P. Chen, X. Liao and T. Manke, Nucl. Phys. Proc. Suppl. 94 (2001) 342.

[7] C. Michael, Proceedings of Heavy Flavours 8, Southampton, UK, 1999.

[8] C.J. Morningstar, M. Peardon, Phys. Rev. D 60 (1999) 034509

[9] T. Yamazaki, R.S. Hayano, K. Itahashi, K. Oyama, A. Gillizer et al., Phys. Lett. B418 (1998) 246.

[10] R. Barth, P. Senger, W. Ahner, P. Beckerle et al., Phys. Rev. Lett. 78 (1997) 4007.

[11] F. Klingl, N. Kaiser and W. Weise, Nucl. Phys. A624 (1997) 527.

[12] A. Sibirtsev, K. Tsushima and A.W. Thomas, Eur. Phys. J. A6 (1999) 351.

[13] K. Seth, Proceedings of Int. Workshop 'Structure of Hadrons', Hirschegg 2001.

[14] W. Greiner, Int. Journal of Modern Physics E, Vol. 5, No. 1 91995) 1-90. 